\title{
Microbiological study of ventilator-associated pneumonia (VAP) after implantation of pneumonia program zero (NZ)
}

\author{
R Fernández Fernández ${ }^{*}$ MR Ramírez Puerta, ME Yuste Ossorio, O Moreno Romero, M Muñoz Garach \\ From ESICM LIVES 2015 \\ Berlin, Germany. 3-7 October 2015
}

\section{Introduction}

The National Survey of Nosocomial Infection Surveillance (ENVIN) is a computerized record of the incidence of nosocomial infection in ICU in Spain. In our hospital, we have drafted rules for the maintenance of the airway in mechanically ventilated patients following general recommendations.

\section{Objectives}

Describe the clinical diagnosis and microbiology of VAP after the implementation of the NZ program.

\section{Methods}

18 ICU beds. Data from ENVIN 01.04.11 / 01.04.15 (clinical diagnosis, microorganisms isolated and antibiotic resistance, inflammatory response ).

NZ: Educational campaign (presencial and by means of internet) to professionals of our unit and anesthesiaresuscitation unit (112 professionals). Were instituted mandatory measures for the prevention of VAP.

\section{Results}

01.04.11/01.04.12- 15 VAP.- Clinical diagnosis:

support clinical radiological+new infiltrate $80 \%$.

Extension of previous infiltrate + clinical worsening (2nd pneumonia)20\%-Germ:

A. baumanii $44.44 \%$ : resistance: amikacin, ampicilin, imipenem, tobramicin

P. aeuroginosa 22.22\%: resistance: amikacin, ceftacidim, ciprofloxacin,

colistin, imipenem, levofloxacin, meropenem, piperacilinatazobactam (P-T)

Hospital Universitario San Cecilio, Unidad Cuidados Intensivos, Granada, Spain

c 2015 Fernández Fernández et al.; This is an Open Access article distributed under the terms of the Creative Commons Attribution License (http://creativecommons.org/licenses/by/4.0), which permits unrestricted use, distribution, and reproduction in any medium, provided the original work is properly cited.
MRSA $16.67 \%$

Polymicrobial $26.67 \%$.

- Inflammatory response: sepsis $33.33 \%$, severe sepsis $33.33 \%$.

01.04.12/01.04.13-6 VAP- Diagn:

support clinical radiological+new infiltrate $66.67 \%$

(2nd pneumonia) $16.67 \%$,

CT 16.67\%.- Germ:

S. Aureus $22.22 \%$ : no resistance

S. Maltophilla $22.22 \%$ no resistance

Polymicrobial $50 \%$.

Without diagnostic $16.67 \%$

- Inflammatory response: sepsis $50 \%$, severe sepsis $33.33 \%$, shock $16.67 \%$

01.04.13/01.04.14- 10 VAP- Diagn:

support clinical radiological+new infiltrate $60 \%$

extension of previous infiltrate + clinical worsening

(2nd pneumonia) 30\%,

CT 10\%.-Germ:

S. Aureus $25 \%$

E. Coli 16.67\%: resistance: ciprofloxacin, amox-clav.

H. Influnzae $8.33 \%$

P. aeuroginosa 8.33\%: resistance :cefepime, ceftaci-

dime, imipenem,

meropenem, P-T

polimicrobial $20 \%$.

- Inflammatory response: sepsis $50 \%$, severe sepsis $30 \%$, shock $20 \%$.

01.04.14/01.04.15- 3VAP

- Diagn:

support clinical radiological+new infiltrate $68.57 \%$

extension of previous infiltrate + clinical worsening

(2nd pneumonia) $22.86 \%$

CT 8.57\%.- Germ: extension of previous infiltrate + clinical worsening 
A. baumanii $18.6 \%$. Resistance: amikacin

P. aeuroginosa $16.28 \%$. Resistance: amikacin, cefepime

S. aureus 13.95\%: no resistance

Polimicrobial $28.57 \%$

- Inflammatory response: sepsis $33.33 \%$, severe sepsis $66.67 \%$

\section{Conclusions}

Most of the diagnoses are made by the patient's clinical and $x$-ray. Each year vary germs being the most frequent $\mathrm{S}$. aureus and P. aeruginosa. Usually, the most common inflammatory response is sepsis. The P. aeruginosa is the germ which has more resistance.

\section{Grant Acknowledgment}

UCI S. Cecilio

Published: 1 October 2015

\section{Reference}

1. [http://hws.vhebron.net/envin-helics/].

doi:10.1186/2197-425X-3-S1-A1021

Cite this article as: Fernández Fernández et al.: Microbiological study of ventilator-associated pneumonia (VAP) after implantation of pneumonia program zero (NZ). Intensive Care Medicine Experimental 2015 3(Suppl 1): A1021.

\section{Submit your manuscript to a SpringerOpen ${ }^{\mathcal{D}}$} journal and benefit from:

- Convenient online submission

- Rigorous peer review

- Immediate publication on acceptance

- Open access: articles freely available online

- High visibility within the field

- Retaining the copyright to your article 